\title{
Organization of work of education volunteers online
}

\author{
A.S. Zelko, and V.S. Maslo \\ Immanuel Kant Baltic Federal University, 236016, Kaliningrad (Russia)
}

\begin{abstract}
This article explorers the concept of online volunteering, the historical background of its emergence and distribution, the current state of this phenomenon. The essence of the project "Online Education Volunteers", which is being implemented on the basis of the IKBFU (Kaliningrad, Russia), is revealed. The project is based on the ideas of the cluster approach in education. The stages of project organization are described in detail. The results of a survey of volunteers regarding their attitude to online education are presented. As a result, risks were foreseen that hinder the introduction of online volunteering. The features of testing the program and the organization of reflexive practices based on the results of the project are revealed. As a result, the general goals of online education were identified, contributing to productive interaction and the main areas of training online volunteers. It is concluded that the implementation of the project makes it possible to create an informationrich educational online environment based on the principles of network interaction and social partnership, to implement a cluster approach to build a new educational space, including further joint development of developmental programs, practices and educational relationships with an emphasis on personal development and self-realization of both schoolchildren and students. The tools proposed in the article stimulated the emergence of some pedagogical precedents, which can become the basis for further research and methodological development.
\end{abstract}

\section{Introduction}

Nowadays, thousands of volunteers around the world carry out volunteer activities, and therefore, there are more and more different directions and forms of such activities, as well as ways of their implementation. New opportunities for volunteering are driving more and more people to volunteer, and those already involved in volunteering can expand their commitments. One of the youngest and most relevant areas of volunteering is online volunteering, which is gaining increasing mass and popularity in connection with the modern realities of the global pandemic and at the same time with a fairly high level of development of information and communication technologies.

Online volunteering is a type of volunteer activity carried out remotely using a device connected to the Internet, through which volunteers are recruited, trained or instructed, volunteer activities are carried out, coordinated and monitored. 


\section{The literature review}

The history of online volunteering dates back to the 1970s, when Project Gutenberg recruited online volunteers to form a public digital library [1]. The Volunteer Match website [2], launched in 1998, claims to have been attended by more than 16.3 million volunteers over its lifetime. More than 131.5 thousand non-profit organizations post their projects and applications on the Volunteer Match platform, and those interested in volunteer work can consider and select applications based on their own interests and capabilities.

Thousands of non-profit organizations and other projects are currently collaborating with online volunteers. The Online Volunteering service, launched in 2000, immediately attracted thousands of volunteers [3]. This service, which links organizations working in developing countries with online volunteers, has statistics that 12 thousand volunteers a year join the service to carry out their activities online, the platform reaches 187 countries, including developing countries (60\% of volunteers), as well as $94 \%$ of volunteers and organizations are satisfied with the results of their collaboration.

In fact, online volunteering consists of a range activities such as translation, conducting research, website design, analysis of data, facilitating or moderating online discussions, database building, writing sentences, writing articles, online mentoring, coaching, tutoring, professional consulting, curriculum development and publication design [4], conducting online projects, translating various documents from one language into another, legal assistance, creating websites to support people in straitened circumstances, creating educational materials for online resources [5], functional or operational activities (technical support, fundraising, communications, marketing, etc.), management consulting and, less commonly, mission-related programming activities (research, writing, editing, leading a discussion group via email, etc.) [6].

The tools used to conduct online volunteering include: emails, instant messaging, discussion groups, social media, and, moreover, phone calls can be effective for managers to facilitate coordination of online volunteers. [7]

Contemporary researchers Danaiata D, Gligor-Cimpoieru DC, Hurbean L, Munteanu V. [7] believe that in the future, the global Internet may provide new opportunities to draw attention to social change and reduce socio-economic gaps between developing and developed countries and as well as individuals.

There is a number of studies devoted to the research of motivation of online volunteers $[8,9]$, the potential of online volunteering $[4,5,7,10]$, older adults' participation in online volunteering [11], the contribution of online volunteering [12], the use of social networks in organizing online volunteer activities [13, 14].

Online volunteering in Russia is still gaining popularity. For example, an online project "Help Map" was created in the abnormally hot summer of 2010. This is an information resource about victims of fires and the collection points for aid. This resource also enables to track cases of deforestation and forest pollution, cases the environmental harm etc.

To search for possible vacancies online volunteering for schoolchildren, there is a Russian-language platform "volunteers of the future". On the platform, you can get acquainted with campaigns held, including in the field of online volunteering, for example, "We are Together" - a support campaign for low-mobility citizens over 60 years old who carry self-isolation. The essence of the campaign is to make regular video calls via Skype or other alternative resources to communicate with people in need. In addition, the platform provides information on volunteering contests "Volunteers of the Future 2020" or "Volunteer of Russia", the campaign "Children instead of flowers", about the work of the charitable foundation "Life as a Miracle". 
Many volunteer centres are converting voluntary work into a distance format: they conduct consultations by phone, webinars and even master classes on teaching foreign languages. Moscow volunteer centre "Silver Age", where volunteers aged 50 and over organize and conduct various trainings, master classes, create intellectual clubs online.

Thus, a master class on the topic of memory preservation for the Boarding House for Labour Veterans was held and recorded, and now the video recording of this webinar is broadcasted on the internal television network of the boarding house. Now all residents of the boarding house can train their memory on a regular basis.

In the field of online education in the context of a global pandemic, volunteer assistance has an unexpected direction - assistance to universities switching to distance learning: within many educational institutions, including the Altai State Technical University (AltSTU), as well as the Higher School of Economics in Moscow intensive work of volunteers to set up digital interaction between students and teachers, support webinars and assist in creation of media content.

\section{The method of the research}

According to UNESCO, in 2020, during a pandemic, more than a hundred countries closed educational institutions nationwide, affecting more than half of the world's students [15]. The impact that the coronavirus pandemic has had on the entire education system differs from country to country, and has its own specifics in different regions of the world. Nevertheless, the general vector of these changes can be traced: the pandemic has put educational organizations in conditions, forcing them to adapt to the events taking place in the shortest possible time, including improving digitalization.

As it stands today there is no universal model that would allow large masses of participants in educational relations to quickly reorganize into an online learning format and return back to offline with minimal losses in the quality of educational results. There is no system of support for these participants [16].

The forced transition to distance education of all educational organizations of general education of the Kaliningrad region in 2020 provoked a number of difficulties. According to the Ministry of Education of the Kaliningrad Region, schoolchildren needed various help:

- additional explanation of the new material;

- help with homework;

- support in the skills of self-organization;

- organization of extracurricular activities;

- solution of technical spectrum issues.

Due to these circumstances, at the Institute of Education of the IKBFU, in partnership with the Ministry of Education of the Kaliningrad Region, a new project "Online Education Volunteers" was launched. The executor of the "Online Education Volunteers" project was the social pedagogical laboratory "Project Incubator" of the Institute of Education of the IKBFU $[17,18]$. When thousands of regional schoolchildren found themselves in a new educational situation, the solutions to the problems listed above were offered.

The essence of the project is to qualitatively update the technologies, forms and content of volunteer assistance from students to teachers in interaction with schoolchildren in an educational online environment. The main idea of the project is related to the enrichment of the online educational environment with innovative content through effective social partnership and a new form of volunteering.

The emergence and successful functioning of such a project is based on the cluster approach. It is a direction in science and practice associated with the search and implementation of new opportunities for the development of society, increasing its well- 
being and competitiveness through the creation and management of clusters. The concept of "cluster" itself means the union of several homogeneous elements, which can be considered as an independent unit with certain properties.

The modern definition of the concept of a cluster, which is quite applicable to the education system, was formulated in the late 1980s by the American economist Michael Porter. In his opinion, "a cluster is a group ... of interconnected organizations operating in a certain area and defined by common activities and complementary to each other" [19].

In this work, we rely on the provisions of Shamova T.I. about the cluster approach. In the definition of a cluster, the following main characteristics can be distinguished:

- there is always more than one element in a cluster;

- all these elements must be homogeneous;

- all these elements work together;

- the work is performed by them more efficiently than by one element;

- the result differs not only quantitatively, but also qualitatively;

- there is a certain criterion by which this efficiency can be assessed.

The main purpose of creating a cluster is to increase the efficiency of the application of any subject or method. Any set of homogeneous elements, jointly performing a common function with greater than a single element efficiency and managed as a whole, can be considered as a cluster.

Thus, the implementation of the project "Online Education Volunteers" is based on the principles of the cluster approach in education. In this case, it is the core of the cluster, which is a collection of representatives of educational organizations of different levels within a given geographic territory, enterprises - suppliers of resources and employers, as well as coordinators and authorities, whose activities are aimed at coordination of the project.

\section{Results}

Volunteering is an integral part of the professional education of a teacher. It is only through such work that the necessary values and attitudes are formed. However, such activities require careful preparation.

The organization of the project consisted of the following stages:

1. Informing about the launch of the project. At this stage, work was organized with schools, which are notified of the opportunities offered by the university. The information stage was implemented both by the regional Ministry of Education and by the university itself.

2. Collecting and analysing the requests of educational organizations of general education. The ministry prepared a document that contained information about school, child and what kind of assistance was required.

3. Before the launch of the project, a volunteer training program was developed in the laboratory, taking into account the online specifics. Psychological and pedagogical support for student volunteers was also organized. The volunteer training program contained instructions, support schemes, communication paths and a reflective map. The program was developed on the basis of the social pedagogical design laboratory "Project Incubator" of the Institute of Education of the IKBFU.

4. Recruitment of volunteers in accordance with the educational needs of schools in the region. At this stage, volunteers were distributed among schools. The curators from the university and the school were assigned, and they meet with parents and schoolchildren.

Among student volunteers was conducted a survey, the purpose of which was to find out the attitude towards online education. The questionnaire covered the preferred forms 
and means of distance learning, the effectiveness of distance learning, discipline regulation in distance learning and the difficulties that volunteers had to face in distance learning.

The study identified the following risks that stand in the way of the implementation of distance education and the disclosure of the creative potential of the volunteer personality in working with schoolchildren:

- the executive position of a volunteer and a decrease in the creative approach to work;

- the volunteers may not be ready for teaching subjects or extracurricular activities;

- volunteers can participate in the work formally and do not show initiative in the implementation of pedagogical innovations;

- a break in communication between all project participants.

5. Approbation of the program. At this stage, the entire network of online interaction is organized: volunteer-teacher, volunteer-parent, volunteer-curator from the university, volunteer-schoolchild.

Fig. 1 represents a scheme of the interaction of all project participants.

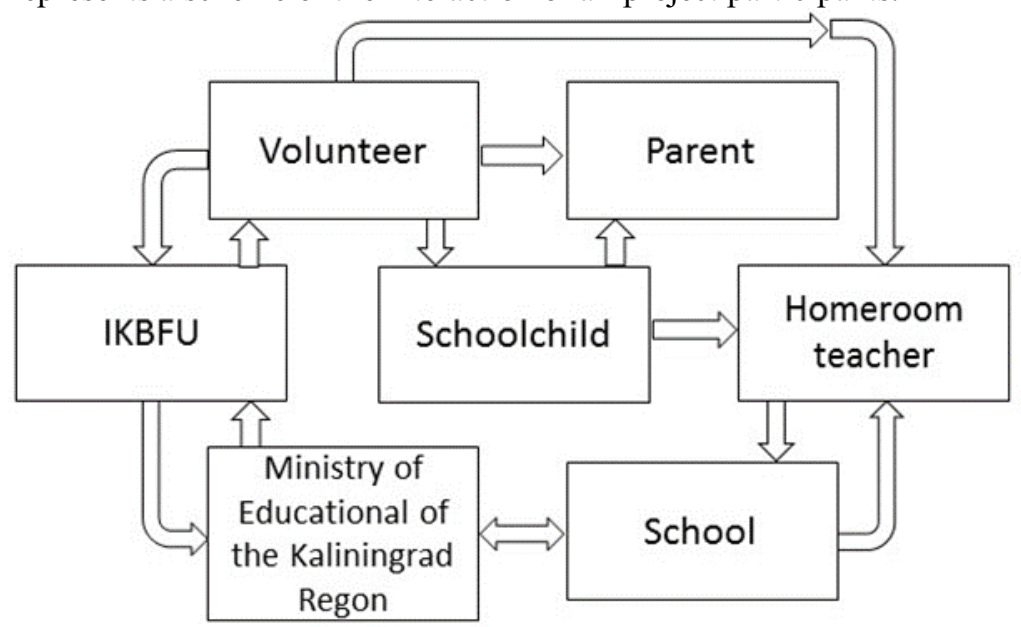

Fig. 1. Scheme of interaction of all project participants

6. Organization of reflective practices based on the results of the project. A sample of the reflexive card is presented in Appendix 1. This form is also a document confirming the activities of the volunteer.

Within the framework of volunteer activities and distance pedagogical practice, students had the opportunity to try themselves in the role of mentors and assistants in organizing the educational process, assistants in the development of educational autonomy of schoolchildren and motivation for learning.

By organizing such volunteering activities, they gain the professional skills they need in the digital age. It is also important to note that they have shown a professional and personal readiness to volunteer by studying online themselves.

As a result of approbation of such activities, we have identified the general goals of online education for both schoolchildren and students, which can contribute to productive interaction:

- development of personal interests in online activities: explore existing personal interests, emergence of new hobbies, analyse their own performance, express and discuss interests, correlate personal interests with the interests of other people;

- search for situations for active personal activity, awakening real interest: identify and explore areas of activity that awaken interest, differentiate areas of activity for different conditions, formulate questions regarding their own actions, explore their own opportunities for cooperation with other people; 
- readiness for action: define and give reasons for actions, taking into account own interests of the activity and existing conditions in practice, define and explain the goals of actions and justify step-by-step actions, represent and discuss the interests of the activity in the group, reflect on the interests of the activity and ability to change them if necessary;

- evaluation of the results of activities: to recognize them, present and discuss them, correlating with the original goals;

- the ability to draw conclusions from the results of the performance assessment: find the reason for the positive and negative assessment results, if necessary, change their own behaviour in accordance with the goals, determine the goals of future activities and combine the educational process with experience.

As you can see, these goals correspond with the essence of the development of the program and its implementation, thus we plan to rely on them in the process of further work.

\section{Discussion}

The main directions of training student volunteers for the further development of the online education system should be:

- leading the programs aimed at increasing digital literacy into the system of training online volunteers of education;

- providing conditions for the development and self-development of an individual in a situation of distant, general and professional culture of online volunteers as bearers of values;

- development and testing of training models for online education volunteers, focused on the ability to work in a distance learning environment in all the variety of types of educational organizations;

- scientific, informational, academic and methodological support of the educational process online;

- creation of a network of information and analytical structures with educational literature and data banks, providing future teachers with methodological and consulting services;

- advanced development of the system of training, advanced training and retraining of pedagogical and managerial personnel in the conditions of distance.

\section{Conclusion}

The implementation of the project allows to create an information-intensive educational online environment, that is based on the principles of network interaction and social partnership. It also offers possibility to implement a cluster approach to build a new educational space, including the joint development of developmental programs, practices and educational relationships with an emphasis on personal development and selfrealization of both schoolchildren and students in the future.

The environment created in this way will enhance the tendencies of individualization of development routes, self-realization, increase the coverage of schoolchildren who have been provided with timely pedagogical support and students who have gained new experience in working with people. By means of the organization of such volunteering activities, the volunteers gain the professional skills they need in the digital age. In addition, the volunteers themselves, while studying remotely, demonstrated the best human qualities: responsibility, professional and personal readiness for social service, which can speak of a high level of development of educational work at the institute in general. 
Thus, the situation described above stimulated the emergence of the following pedagogical precedents, which can become the basis for further research and methodological development:

- features of educational situations in which each student can act differently, depending on his character and motivation;

- contradictions in the content of pedagogical activity, provoked by the situation of online education;

- individual framework of communication and specific conditions for the interaction of all participants in educational relations.

\section{References}

1. J. Cravens, Nonprofit Quarterly 14, 65-68 (2007).

2. Volunteer Match Homepage, https://www.volunteermatch.org/

3. UNV Online Volunteering service Homepage, https://www.onlinevolunteering.org/en

4. J. Cravens, The International Journal of Volunteer Administration 26 (1), 15 - 23 (2006).

5. Y. Amichai-Hamburger, Computers in Human Behaviour 24 (2), 544 - 562 (2007).

6. B. Bezmalinovic Dhebar, B. Stokes, Nonprofit Management and Leadership 18 (4), 497-506 (2008).

7. D. Danaiata, D.C. Gligor-Cimpoieru, L. Hurbean, V. Munteanu, Paper Presented at the 7th International Management Conference "New Management for the New Economy", 174-186. Bucharest, Romania (2013).

8. A. Baruch, A. May, D. Yu, Comput Human Behav 64, 923-931 (2016).

9. J. Cox, E.Y. Oh, B. Simmons, G. Graham, A. Greenhill, C. Lintott et al., Doing Good Online: an Investigation into the Characteristics and Motivations of Digital Volunteers (2015)

10. N.M. Pestereva, O.S. Nadeina, S.L. Aleksandrovna, International and Russian experience of volunteer activity: history, modern situation and future prospects 11 (10), 378-388 (2014).

11. D. Mukherjee, Ageing Int 36 (2), 253-266 (2010).

12. A.C. Powell, K. Casey, D.J. Liewehr, A. Hayanga, T.A. James, G.S. Cherr, J Am Coll Surg 208, 304-312 (2009).

13. S. Conroy, A. Wiliams, Use of Internet, Social Networking Sites, and Mobile Technology for Volunteerism: Implications for Volunteer Recruitment and Engagement (AARP, Washington, DC, 2014).

14. R.J. Raja Yusof, A.A. Norman, S.S. Abdul Rahman, N. Nazri, Z. Mohd Yusoff, Computers in Human Behavior 57, 388-397 (2016).

15. UNESCO Homepage, https://en.unesco.org/

16. A.O. Budarina, A.S. Zelko, Management of social projects in higher educations: setting objectives for implementing educational activities, The European Proceedings Social and Behavioural Sciences EpSBS XLV, IFTE 2018 - 4th International Forum on Teacher Education, pp. 1-7. The Future Academy, Kazan, Russia (2018).

17. A.O. Budarina, A.S. Zelko, Pedagogical Strategies for the Development of Soft Skills of Schoolchildren in After Class Education, IFTE 2020 - 6th International Forum on Teacher Education, ARPHA Proceedings, Kazan, Russia (2020). 
18. A.S. Zelko, The role of volunteerism in the professional upbringing of students, International Conference "Process Management and Scientific Developments", Scientific publishing house Infinity, Birmingham, United Kingdom (2020). 